\title{
DERECHOS INDIVIDUALES Y DEBERES DE ESTADO. CONSIDERACIONES BIOÉTICAS EN TORNO AL DEBATE SOBRE LA ANTICONCEPCIÓN DE EMERGENCIA EN CHILE
}

\author{
Raúl Villarroel Soto*
}

\begin{abstract}
Resumen: El presente artículo intenta dar cuenta de un debate de relevancia moral, social y política en Chile: la controversia acerca de la anticoncepción de emergencia y la iniciativa de las autoridades de gobierno de facultar a los servicios de salud del Estado para distribuir el fármaco comúnmente llamado "píldora del día después". Se abordan diferentes perspectivas de análisis y se recae, finalmente, en un examen bioético a la luz de una revisión de la perspectiva principialista clásica para advertir algunas de sus insuficiencias analíticas y, por lo mismo, resituar la problemática desde la perspectiva de los derechos humanos.
\end{abstract}

Palabras clave: bioética, anticoncepción de emergencia, píldora del día después, principialismo bioético, derechos humanos, deberes de Estado

\section{INDIVIDUAL RIGHTS AND STATE DUTIES, BIOETHICAL CONSIDERATIONS AROUND THE DEBATE OVER EMERGENCY CONTRACEPTION IN CHILE}

\begin{abstract}
This essay tries to show a moral, social and politically relevant debate in Chile: the controversy about emergency contraception and the decision of government authorities to authorize state health care services to distribute the drug commonly known as "the day after pill". Different views of analysis are studied to reach, finally, a bioethical reflection of the problem considering a re-examination of the classical principles perspective, to point out some of its analytical inadequacies and, for this reason, to rethink the problem under a human rights perspective
\end{abstract}

Key words: bioethics, emergency contraception, the day after pill, bioethics principles, human rights, state duties

\section{DIREITOS INDIVIDUAIS E DEVERES DE ESTADO, CONSIDERAÇÓES BIOÉTICA SOBRE O DEBATE A RESPEITO DA ANTICONCEPÇÃO DE EMERGÊNCIA NO CHILE}

Resumo: O presente artigo tenta apresentar um debate de relevância moral, social e política no Chile: a controvérsia em torno da anticoncepçáo de emergência e a iniciativa das autoridades de governo de facultar os serviços de saúde do Estado para distribuir o fármaco popularmente conhecido como "pílula do dia seguinte". Abordam-se diferentes perspectivas de análise e se fixa, finalmente, num exame bioético do problema, à luz de uma revisão da perspectiva principialista clássica, para alertar a respeito de algumas de suas insuficiências analíticas e por, isto mesmo, recolocar a problemática a partir da perspectiva dos direitos humanos.

Palavras-chave: bioética, anticoncepção de emergência, pílula do dia seguinte, principialismo bioética, direitos humanos, deveres de Estado

Doctor en Filosofía y Magíster en Bioética. Centro de Estudios de Ética Aplicada, Facultad de Filosofía y Humanidades, Universidad de Chile, Chile Correspondencia: rvillarr@uchile.cl 


\section{Presentación ${ }^{1}$}

A menudo se habla de derechos sexuales y reproductivos por referencia a aquella garantía básica de parejas e individuos para decidir de manera libre y responsable acerca del número de hijos que desean tener y el intervalo entre los nacimientos; por cierto, contando con la información, la educación y los medios para poder hacerlo. Se trata de un hecho complejo en el que se articulan funcionalmente, además, varios otros derechos: a la salud, a la libertad individual, a la libertad de pensamiento, de conciencia y religión, de opinión y expresión, a la información y educación, a los beneficios del progreso científico, entre otros.

De cara al resguardo y cumplimiento de estos mismos derechos, el Estado chileno ha adherido a una serie de pactos internacionales derivados de algunas conferencias de Naciones Unidas, en particular la de Población y Desarrollo, de El Cairo, llevada a cabo en 1994, así como la IV Conferencia Mundial de la Mujer, realizada en Beijing en 1995.

Por eso, cuando emerge un debate como el que ha tenido lugar en Chile durante el último tiempo, a propósito de la disposición del Ministerio de Salud para distribuir gratuitamente el anticonceptivo de emergencia llamado "píldora del día después" a jóvenes mayores de 14 años, sin un expreso consentimiento o conocimiento de sus padres o representantes, pareciera que nos hallamos frente al siempre controversial tema de los límites y la extensión del ejercicio de derechos por parte de las personas; en este específico caso, el de sus derechos sexuales y reproductivos, y de los deberes que al Estado conciernen en procura de su protección.

Parece conveniente examinar primero esta situación desde algunas de sus dimensiones -históricas, científicas, jurídicas, políticas, religiosas-, para luego considerar bioéticamente los principios y derechos en juego a la hora de decidir sobre la legitimidad de la medida oficial que ha dado lugar a esta controversia.

\section{La dimensión histórica}

En 1964, el Ministerio de Salud implementó un programa de planificación familiar inicialmente denominado "Salud Familiar y Regulación de los Nacimientos" y que pronto pasó a llamarse "Programa de Paternidad

Una versión anterior del presente trabajo fue presentada para publicación en Perspectivas Éticas. No 14. Universidad de Chile. 2006.
Responsable"(1). Con ello se pretendió enfatizar el derecho de las parejas a decidir libremente cuántos hijos querían procrear; su objetivo final era disminuir la incidencia de abortos provocados reduciendo, de paso, los riesgos involucrados para la salud maternoinfantil. Posteriormente, a partir de 1971, el principio de regulación de la natalidad se integró de lleno a las políticas sanitarias gubernamentales, extendiéndose su cobertura a casi la mitad de la población en edad de procrear.

En 1979, y como consecuencia del sesgo ideológico pronatalista impuesto a partir del golpe militar del año setenta y tres, la Oficina de Planificación Nacional del gobierno de la época formuló su "Política de Población", en la que abogaba claramente por una restricción del uso de métodos anticonceptivos, cuestión que se mantuvo a lo largo de todo el período. Sólo en la década de los 90, una vez recuperada la democracia, se vuelve a reconsiderar la perspectiva de análisis y se estipulan -mediante su última versión de 1993- las "Normas de Paternidad Responsable" por parte del Ministerio de Salud.

En 1997, la Salud Sexual y Reproductiva (concepto que reemplazó al de "control demográfico", a partir de la Conferencia Internacional sobre Población y Desarrollo, celebrada en El Cairo, en 1994) fue definida como una de las 16 prioridades de salud para el país y en el Programa de Salud de la Mujer dicha actividad pasó a llamarse "Control de Regulación de Fecundidad".

Entre otros métodos anticonceptivos, incluyendo reversibles e irreversibles -exceptuándose el aborto, alternativa prohibida legalmente en Chile-, se incluye a la píldora postcoital, o "píldora del día después", como se le conoce comúnmente, método que hace algunas décadas no se encontraba disponible en el país, pese a ser conocido desde la década de los sesenta. De acuerdo con estudios realizados por la Organización Mundial de la Salud (OMS), es el método anticonceptivo de emergencia más efectivo y mejor tolerado. Las píldoras para la anticoncepción de emergencia (PAE) son eficaces e inocuas y no tienen contraindicaciones médicas.

En nuestro país, la autorización en 2001 para comercializar Postinal, un producto destinado a la anticoncepción de emergencia, provocó innumerables debates, debido a la firme oposición de los grupos autodenominados "pro vida" y la jerarquía de la Iglesia Católica, que la consideran abortiva. Ya en 2004 comienza por 
primera vez a distribuirse gratuitamente en los diferentes servicios de salud de la capital, en virtud de una disposición ministerial, la "píldora del día después".

La historia culmina, hasta donde podemos dar cuenta de ella, con la disposición reciente que faculta a los servicios de salud para entregar el anticonceptivo de emergencia a la población mayor de 14 años que lo requiera, sin conocimiento o consentimiento expreso de sus padres. Las circunstancias en que aquello tiene que ocurrir es asunto que explicitaremos posteriormente, porque aporta elementos de juicio para una consideración más pormenorizada y significativa de los hechos.

\section{La dimensión biomédica}

En un boletín informativo de marzo de 2005(2), la OMS señaló que la anticoncepción de emergencia se refiere a aquellos métodos que las mujeres pueden usar dentro de los primeros días posteriores a una relación sexual sin protección, con el objetivo de prevenir un embarazo no deseado, dejando expresa constancia que esta clase de métodos anticonceptivos no son adecuados para uso regular. El régimen de anticoncepción de emergencia recomendado por la OMS es 1,5 mgs. de levonorgestrel administrado en una sola dosis y está dirigido a toda mujer en edad reproductiva que pudiera necesitarlo.

Según el documento, la anticoncepción de emergencia está pensada para situaciones en las que, por ejemplo, no se ha usado ningún método anticonceptivo o ha ocurrido una falla del método o éste ha sido usado de manera incorrecta. La última circunstancia que el documento menciona para justificar su empleo es cuando la mujer ha sufrido una agresión sexual sin estar protegida con un procedimiento anticonceptivo eficaz.

El informe asegura que se encuentra demostrado que las PAE que contienen levonorgestrel previenen la ovulación y carecen de un efecto detectable sobre el endometrio (revestimiento interno del útero) o en los niveles de progesterona cuando son administradas después de la ovulación. Es claro que las PAE no son eficaces una vez que el proceso de implantación se ha iniciado y no provocarán de manera alguna un aborto.

También se hace hincapié en que las PAE no deben ser administradas si lo que se pretende es interrumpir un embarazo confirmado porque ya resulta ser demasiado tarde para ello. Aunque pareciera haber consenso por parte de los expertos de que no hay daño eventual para la mujer o para el feto si se usan píldoras anticonceptivas de emergencia inadvertidamente durante las primeras semanas de embarazo.

La recomendación indica claramente que las PAE deben ser usadas únicamente en casos de emergencia y no resultan apropiadas si se emplean como método anticonceptivo de uso regular, porque hay mayor probabilidad de que fallen en comparación con los anticonceptivos modernos. Además, el uso frecuente de anticoncepción de emergencia tiene como resultado efectos secundarios, tales como irregularidades menstruales. No obstante, el uso repetido no conlleva riesgos conocidos hasta ahora para la salud.

\section{La dimensión jurídica}

En Derecho Internacional, los Estados se obligan jurídicamente ante la comunidad mundial cuando suscriben tratados globales. Los mismos pueden también transformarse en obligaciones para el orden jurídico interno cuando a éste se incorporan las normas de aquellos tratados suscritos. De esto se puede deducir que las obligaciones que emanan de dichos tratados son, por una parte, internacionales y, por otra, nacionales, debiendo el Estado responder por cada una de ellas ante las instancias que corresponda(3).

Los Derechos Humanos se consideran parte del derecho internacional y han sido aceptados y reconocidos por los Estados suscriptores como "derechos inherentes a, o de la esencia de, la persona humana”. Basándose en el principio de no discriminación, los Estados tienen el deber de garantizar y asegurar el acceso a estos derechos a todas las personas. $\mathrm{Al}$ amparo de este principio se despliega la positivización del derecho a la salud reproductiva de todas las personas, por ejemplo.

En este sentido, la expresión "discriminación contra la mujer" está definida en el Artículo $1^{\circ}$ de la comúnmente llamada Convención de la Mujer(4), como “...toda distinción, exclusión o restricción basada en el sexo que tenga por objeto o por resultado menoscabar o anular el reconocimiento, goce o ejercicio por la mujer, independientemente de su estado civil, sobre la base de la igualdad del hombre y la mujer, de los derechos humanos y las libertades fundamentales en las esferas política, económica, social, cultural y civil o en cualquier otra esfera". 
En suma, los tratados internacionales jurídicamente vinculantes y que, además, han sido promulgados en Chile tienen fuerza obligatoria en el territorio nacional. El Estado chileno debe propiciar que estas obligaciones se cumplan y que los objetivos planteados por estos instrumentos no sean obstaculizados por ninguno de los otros órganos o poderes del Estado, e incluso por particulares. Estos derechos pueden, a la vez, ser invocados por las personas en forma directa o indirecta.

Entre las obligaciones internacionales señaladas en los documentos oficiales está el derecho a la salud. En la definición establecida por la OMS en su Constitución (1946) se afirma que su contenido es complejo y está referido al estado de completo bienestar físico, mental y social, y no solamente a la ausencia de afecciones o enfermedades. El Estado tiene entonces un doble deber: promover la salud y prevenir o eliminar todas las barreras que obstaculicen las posibilidades de las personas para alcanzar ese mínimo estándar reconocido internacionalmente.

A su vez, la Declaración Universal de los Derechos del Hombre de 1948 y su antecedente, la Carta de las Naciones Unidas de 1945, plantean en forma genérica los compromisos que adoptaron las naciones, en el sentido de promover mejores condiciones de vida, desarrollo social y económico en áreas tales como la salud. En un sentido mucho más amplio, el derecho a la salud también se encuentra implícito en la Convención Americana sobre Derechos Humanos (Pacto de San José). Estos derechos se encuentran, a su vez, consagrados en el Pacto Internacional de Derechos Civiles y Políticos, ratificado por Chile en 1972, en el cual se entiende que para gozar y tener garantías efectivas del derecho a la vida es esencial-además de no ser sometido a tratos crueles o inhumanos o a la pena de muerte- disponer del derecho y de la garantía efectiva a la salud (art. 6.1).

En este contexto, la Convención de la Mujer reafirma el derecho a la salud y hace particularmente obligatorio adoptar resoluciones que eliminen toda forma de discriminación en el área de la anticoncepción cuando señala: "Los Estados Partes adoptarán todas las medidas apropiadas para eliminar la discriminación contra la mujer en la esfera de la atención médica, a fin de asegurar, en condiciones de igualdad entre hombres y mujeres, el acceso a servicios de atención médica, inclusive los que se refieren a la planificación de la familia" (4, Art. № 12.1). Posteriormente, en su artículo
16.1 señala: "Los Estados Partes adoptarán todas las medidas adecuadas para eliminar la discriminación contra la mujer en todos los asuntos relacionados con el matrimonio y las relaciones familiares y, en particular, asegurarán condiciones de igualdad entre hombres y mujeres", especificando en su letra e) que se trata de "Los mismos derechos a decidir libre y responsablemente el número de sus hijos y el intervalo entre los nacimientos, y a tener acceso a la información, la educación y los medios que les permitan ejercer estos derechos" (4, Art. No 16.1 e).

\section{La dimensión política}

Tal como se señala en la Introducción de la guía ministerial para la atención a víctimas de violencia sexual (5), el Estado chileno ha asumido efectivamente el imperativo ético de enfrentar la violencia sexual y disminuir los daños y las secuelas que ésta produce en las personas más vulnerables, materializando con ello el respeto irrestricto de los múltiples compromisos internacionales que ha suscrito y su decisión de implementar efectivamente la nueva legislación promulgada al respecto, en procura de la cual se realizan grandes esfuerzos para adecuar, mediante un proceso de reforma, el Sistema de Salud a las necesidades de la población y, al mismo tiempo, consolidar la estructuración de la Reforma Procesal Penal.

Como todo parece indicar, los esfuerzos se han concentrado en la atención de urgencia, porque es una de las instancias de la red de servicios y de apoyo social que representa la puerta de entrada frecuente para las personas afectadas, y, a menudo, es la única capaz de detectar la situación de violencia. El objetivo es integrar los aspectos propiamente asistenciales de la atención con los judiciales y psicosociales, de modo de evitar que la agresión o abuso se repitan y reparar los daños, hasta donde sea posible.

Por otra parte, las normas nacionales sobre regulación de la fertilidad(6) establecen que: "En nuestro país existe, desde los inicios de la historia de la salud pública, una tradición de respeto a la responsabilidad del Estado en orden a promover políticas y actividades que favorezcan la salud y el bienestar de las personas y las familias, en particular de las más vulnerables. Una de estas políticas ha sido la oferta de servicios de regulación de la fertilidad en el sistema público de salud. Actualmente, en el contexto de una visión de salud integral, estos servicios permiten armonizar la fertilidad humana 
con las necesidades de bienestar y salud de las mujeres y de sus hijos e hijas, brindando a todas las personas la oportunidad de decidir de manera libre e informada respecto a su reproducción" $(6, p .7)$.

En consecuencia, reducir la inequidad reproductiva generada por las desigualdades de género y la mayor vulnerabilidad de algunos grupos de población (de menor nivel socioeconómico, de pueblos originarios, adolescentes y adultos jóvenes); disminuir la incidencia del aborto inseguro provocado, mejorando la oferta y la calidad de los servicios de salud sexual y reproductiva, focalizando los esfuerzos en las mujeres con mayor riesgo de interrumpir voluntariamente su embarazo en condiciones de inseguridad, y aminorar el embarazo no deseado en adolescentes, quienes, por su mayor vulnerabilidad, requieren de servicios apropiados y sin barreras de acceso, se convierte en una tarea de Estado.

Los objetivos sanitarios nacionales para la primera década del siglo XXI plantean continuar disminuyendo la mortalidad materna mediante la reducción de los embarazos no deseados y de alto riesgo. Lograrlo equivale a disminuir la brecha entre fecundidad deseada y fecundidad real, lo que implica focalizar acciones en la población adolescente, fundamentalmente en aquellos sectores de población con menores ingresos.

¿Por qué un proyecto social de esta naturaleza, que intenta perfilarse desde una orientación semejante a la que aquí se ha tratado de describir, ha encontrado resistencia por parte de algunos segmentos de la población, ante la decisión oficial de distribuir el anticonceptivo de emergencia? ¿Por qué iniciativas aparentemente tan bien inspiradas, tan cautelosas de una perspectiva de derechos de las personas y que parecieran expresar un compromiso tan decidido de parte del Estado para intentar resolver problemas de salud pública, justicia y equidad social, conforme a modelos científicos y opciones éticas, no aúnan la voluntad general de la población, y frente a ellas algunos sectores reaccionan como si vieran comprometidas sus opciones de vida buena y amenazados sus valores más preciados?

\section{La dimensión religiosa}

El Arzobispo de Santiago, Cardenal Francisco Javier Errázuriz, en una carta distribuida el 2 de mayo de 2004 en todas las parroquias y capillas de la capital, respecto de la píldora del día después señalaba: "una cosa es segura, se trata de un fármaco contrario a la concepción (...) Ya este hecho no es irrelevante para la conciencia. Sin embargo, la pregunta que ha desatado la gran polémica es otra: ¡elimina o no elimina una vida humana? Si la eliminara, poco importa el respeto que algunos querrían dar a la exaltación de la libertad individual".

Y agregaba: "La distribución gratuita de un fármaco cuya finalidad no es sanar, es un hecho más que controvertido. Peor aún, cuando se hace con dineros aportados por todos los contribuyentes (...) El ejercicio de la propia libertad tiene un límite infranqueable: el derecho a la vida de los demás (...) Vulnera gravemente este principio esa corriente que pretende justificar el aborto como un derecho de la mujer a tomar decisiones sobre su propio cuerpo (...) El niño por nacer ya no es "su cuerpo", es una vida humana distinta a la suya, de la cual no puede disponer (...) Es un gravísimo error y una irreparable injusticia exigir la aprobación de presuntos 'derechos sexuales y reproductivos' que incluyan el derecho al aborto".

Sin necesidad de seguir ilustrando esta misma aproximación, en las palabras de Monseñor Errázuriz se trasunta con claridad el motivo central de la divergencia entre las posiciones sustentadas por las autoridades de gobierno y las organizaciones de la comunidad que las apoyan, por una parte, y las de la Iglesia Católica y los sectores afines a su posición inspirados en la doctrina magisterial, por la otra.

Desde el sector eclesiástico, las objeciones de moralidad al empleo de anticonceptivos, particularmente a aquellos considerados de emergencia, se fundamentan, principalmente, en lo que, desde tal perspectiva, se consideran certezas innegables. Por ejemplo, en que: "El amor, que se alimenta y se expresa en el encuentro del hombre y de la mujer, es don de Dios (...) El hombre, en efecto, es llamado al amor como espíritu encarnado, es decir, alma y cuerpo en la unidad de la persona. El amor humano abraza también el cuerpo y el cuerpo expresa igualmente el amor espiritual. La sexualidad no es algo puramente biológico, sino que mira a la vez al núcleo íntimo de la persona. El uso de la sexualidad como donación física tiene su verdad y alcanza su pleno significado cuando es expresión de la donación personal del hombre y de la mujer hasta la muerte. Este amor está expuesto sin embargo, como toda la vida de la persona, a la fragilidad debida al pecado original y sufre, en muchos contextos socio- 
culturales, condicionamientos negativos y a veces desviados y traumáticos. Sin embargo, la redención del Señor ha hecho de la práctica positiva de la castidad una realidad posible y un motivo de alegría, tanto para quienes tienen la vocación al matrimonio -sea antes y durante la preparación, como después, a través del arco de la vida conyugal-, como para aquellos que reciben el don de una llamada especial a la vida consagrada (...) Con palabras del Concilio Vaticano II, el Catecismo de la Iglesia Católica recuerda que 'Los jóvenes deben ser instruidos adecuada y oportunamente sobre la dignidad, tareas y ejercicio del amor conyugal, sobre todo en el seno de la misma familia"'(7).

Del documento recién citado se puede inferir que la sexualidad es un acto esencialmente conyugal y que sólo bajo tal condición se alcanzan sus fines más preciados: el amor de los esposos y la procreación, expresión más elevada de ese amor y el cumplimiento efectivo del servicio a la vida a que los seres humanos están convocados por Dios. Se podría deducir que la anticoncepción introduciría un elemento de distorsión antinatural en aquel curso de acontecimientos que, a modo de plan divino, está trazado para el hombre; más aún, en el caso de los jóvenes, que debieran estar preparados por la propia familia para aproximarse al cumplimiento de tal designio y no dar curso a semejantes desviaciones.

En su declaración, el Cardenal Errázuriz sostenía a propósito de la píldora del día después: "Los laboratorios que la comercializan ya informan que uno de sus efectos puede ser el de impedir la implantación del óvulo fecundado en el útero materno, produciéndose así la pérdida de una vida humana. Pues bien, cuando se trata de la vida humana, no es lícito emprender una acción sin tener la seguridad de que esa acción no la mata. Como se dice gráficamente, no se puede disparar contra un matorral cuyas ramas se mueven, ignorando si el movimiento lo provoca una liebre o un niño. Por eso, mientras no se llegue a la conclusión contraria, moralmente no es lícito su consumo".

Esta resistencia, inspirada en la lógica del buen cazador, ha tenido asidero en algunos reclamos provenientes de parte de la comunidad científica, que cree haber demostrado, a partir de ciertas investigaciones, el efecto abortivo del levonorgestrel. Un caso significativo sería el estudio llevado a cabo en 2004 por la Facultad de Medicina de la Pontificia Universidad Católica de Chile, a petición del Instituto de Salud Pública.
El informe elaborado por académicos de esa casa de estudios(8) concluyó que, si bien todos los estudios epidemiológicos realizados hasta esa fecha tenían limitaciones metodológicas (falta de grupo control tratado con placebo y estimación poco precisa del momento de la ovulación), las investigaciones habían demostrado la efectividad del levonorgestrel ingerido el día de la ovulación o días después de ésta, y que ello no se podía explicar por otro mecanismo que no fuera el antiimplantatorio. Es decir, la sustancia tenía un claro efecto abortivo. Conjuntamente, el Centro de Bioética de la misma universidad destacó el valor del respeto por la vida humana, sea cual fuere el estado de desarrollo de ésta.

En fecha más reciente, en una declaración pública entregada en Concepción el 6 de septiembre de 2006, la Universidad Católica de la Santísima Concepción afirmó que "toda iniciativa que se relacione con la defensa de la vida, los derechos de la familia y el respeto de la infancia y la juventud, como la recién anunciada por el Ministerio de Salud, requiere de un acuerdo social amplio que la legitime. Nos parece que la aplicación de métodos que pueden derivar en prácticas abortivas para disminuir los embarazos no deseados representa un flagrante atentado contra la vida y una imposición inaceptable por parte del Estado a las personas". Más adelante, en parte de esta misma declaración, se agregaba: "Creemos que los embarazos no deseados en la población juvenil y de escasos recursos constituyen un problema país. Pero las inequidades sociales no se resuelven arriesgando la vida por nacer ni promoviendo una sexualidad precoz, sin calidad afectiva y fuera del ámbito de la familia (...) De haber acciones ilegales contra la infancia y la adolescencia, de las que más tarde se derivara un embarazo, lo que corresponde es investigar las responsabilidades legales y no legitimar estas conductas por la vía de una solución abortiva, como lo es la píldora del día después".

\section{La dimensión bioética}

Lo que está en juego en la discusión actual es, en un sentido, la definición y defensa de determinadas opciones de moralidad, la adhesión a particulares visiones de vida buena, como también se podría decir; en otro, la interpretación de datos provenientes de la investigación científica, que se consideran más o menos concluyentes, en función de determinados supuestos o hipótesis que se tienen por probables y que se esgrimen 
como fundamento de aquellas opciones de moralidad que se busca defender.

Se podrían hacer algunos comentarios desde la perspectiva del así llamado "enfoque principialista”, entendiéndose que cuando hablo de "principios" me refiero a aquellos que fueron enunciados (luego del Informe Belmont) por Beauchamp y Childress(9).

A partir de los planteamientos del bioeticista español Diego Gracia, parece necesario establecer una jerarquía entre esos principios(10), un orden que permita definir cuál de ellos deberá preponderar en los casos de conflicto suscitados frecuentemente en la coexistencia de los individuos, cada vez que éstos traen al espacio público sus reivindicaciones particulares con pretensiones de validez.

Gracia intenta fundamentar aquella jerarquía recurriendo al reconocimiento de dos premisas básicas de marcada inspiración kantiana que, al ser tenidas en cuenta como ejes del análisis ético, permiten legitimar la opción por uno u otro principio, según sea el caso. La primera está referida a la condición de "persona", inherente a todo ser humano, y al hecho de que por ella se le pueda atribuir a éste una dignidad; la otra, a la igualdad entre los hombres y, por ello, al sentido universal de respeto con que éstos deben ser considerados. La primera de estas premisas tendría un carácter ontológico, definiría el ser del hombre; la segunda, un carácter ético, definiría un deber ser para el hombre. Ambas constituirían, por su carácter a priori, un verdadero "sistema de referencia moral" desde el cual sería posible establecer sucesivas y diversas aproximaciones a la realidad humana, encarnadas en las distintas formulaciones o cánones históricos que han surgido a la luz de estos principios formales.

De acuerdo con lo anterior, el enfoque principialista de Beauchamp y Childress daría cuenta de la complejidad de las situaciones dilemáticas de la moralidad contemporánea; sin embargo, cuando los principios entran en conflicto, es posible recurrir a la distinción establecida por David Ross entre "deberes prima facie" (prima facie duties) y "deberes reales" (actual duties)(11). Mediante su distinción, Ross postula una igualdad preliminar de rango o jerarquía entre los principios, lo que permitiría suponer que éstos generan idéntica obligatoriedad, aunque sólo hasta que no entren en conflicto mutuo y nos veamos forzados a atender sólo a uno de ellos y posponer los demás. Al hecho de que en un comienzo todos los principios obligarían de manera semejante, Ross lo denomina su carácter prima facie. Pero lo harían así sólo hasta que la contradicción que ha suscitado esta especie de equilibrio inmovilizador de su fuerza imperativa inicial conmine a otorgar prevalencia a tan sólo uno de ellos, en desmedro de los demás.

Mediante este mismo análisis, Diego Gracia clasifica los principios según si responden a "mínimos" o "máximos" de moralidad(12). El nivel de "mínimos" estaría conformado por los principios de no maleficencia y de justicia, y el de "máximos" por los de autonomía y beneficencia. Como se sabe, los primeros definen "deberes perfectos", vale decir, obligaciones que podrían, incluso, ser impuestas por la fuerza de la ley; los segundos, en cambio, definen más bien "obligaciones morales", es decir, constituyen "deberes imperfectos" que sólo pueden ser sugeridas, mas nunca impuestas; son aquellos deberes cuyo imperativo de cumplimiento emana sólo de la libre voluntad. Los deberes de justicia y no maleficencia son, según esto, perfectos; a saber, deben ser exigidos, aun forzadamente, a todos por igual. Por el contrario, los deberes de beneficencia y de autonomía serán imperfectos porque, estando su cumplimiento remitido a la gestión individual, no pueden ser impuestos al conjunto de la sociedad y sólo el sujeto puede imponérselos voluntariamente a sí mismo, si así lo quiere. Por ello, los deberes perfectos tienen un carácter público y le corresponde al Estado su gestión; en tanto que los imperfectos, teniendo carácter privado, son de exclusiva competencia individual.

Tanto la objeción general al uso de anticonceptivos sostenida en la doctrina de la Iglesia Católica como la resistencia al dictamen ministerial que instruía a los servicios de salud para distribuir la píldora del día después a mujeres mayores de 14 años estarían dentro de las demandas referidas a "máximos" de moralidad. Es decir, se trataría de pretensiones de validez propias del nivel de las ideas de perfección y felicidad, de los sentidos y concepciones particulares de vida buena, o de criterios específicos de virtud. Todas ellas representarían la defensa de convicciones provenientes de códigos de validez y legitimidad restringida a círculos confesionales en los que se comparten tales ideales y visiones.

Parece claro que, tras la negativa a admitir como legítima la decisión de las autoridades sanitarias por parte de los sectores vinculados a la Iglesia Católica, se aloja una concepción filosófica de base y alcance mucho 
más extendido que la simple diatriba en contra de la píldora a que ha dado lugar. Ella dice relación con una específica manera de interpretar la evidencia de la que se ha premunido, a partir de una cierta orientación de la investigación científica que le ha sido propicia. A partir de allí se ha permitido alentar consideraciones metafísicas acerca del estatuto ontológico del embrión humano y del momento en que se puede decidir el comienzo efectivo de la vida humana.

Una perspectiva de tal naturaleza ${ }^{2}$ correspondería a expresiones provenientes de convicciones y adhesión a "máximos" de moralidad, ya que pretenden fortalecer opciones que no podrían ser más que "aconsejadas" o "sugeridas" a los individuos que coexisten en condiciones de "extrañeza moral" en las sociedades contemporáneas.

Por existir un margen difuso e ignoto en el saber exacto de la ciencia respecto de cuestiones como las que en nuestro país se han discutido durante el último tiempo, el debate tiende a centrarse en cuestiones de convicción, en la defensa de máximos de moralidad, en la protección de valores sectoriales preferentemente, por lo cual se tienden a desatender los mínimos necesariamente compartidos y exigibles a todos los individuos.

Aunque el modelo principialista ha tenido éxito al dotar de cierta unidad a las racionalidades escindidas (científico-biológica, económica, social, política, ética) y poner en diálogo los distintos discursos operantes en las cuestiones de salud, adolece en el fondo de ostensibles dificultades. Una de las más importantes se refiere al convencionalismo moral que intenta hacer prevalecer ante la falta de una adecuada fundamentación(13), porque carece del soporte teórico para fundamentar cada principio, en tanto todos ostentan idéntico rango o valor inicial. Ello puede propiciar circunstancias eventuales en las que los más elementales deberes de justicia, por ejemplo, pudieran verse opacados por la primacía de otros deberes funcionales a lógicas de poder o a hegemonías doctrinarias. Cuando se considera al margen de otras concepciones, el modelo principialista

\footnotetext{
Según la cual resulta posible sostener planteamientos tales como: "Siempre quisiéramos respetar a las autoridades civiles, pero a veces nos sentimos obligados a reflexionar si han decidido lo que es justo a los ojos de Dios, ya que, de lo contrario, tenemos que optar por la obediencia a Dios y no por la obediencia a los hombres", u otros como éste: "El rechazo del agresor (en caso de violación) y de cuanto es pertenencia suya no puede justificar la eliminación de lo que nunca le perteneció: de una vida nueva e inocente que quiere nacer".
}

puede dar lugar a ciertas comprensiones de la moral sólo en apariencia universalistas, puesto que a poco andar se advierte su complacencia con determinados intereses particulares, perdiéndose de vista el horizonte de lo humano y el sentido de justicia que le es inherente.

Podría decirse que el modelo de Beauchamp y Childress reproduce en su esencia la pulsión axiomatizante del modelo cientificista de pensamiento y culmina convirtiendo a la moralidad en una pura tecnología de aplicación de recursos, como si los principios de la moralidad pudieran manipularse con la misma pretensión de objetividad con que se manejan los datos empíricos. Este modelo carece de la profunda reflexividad que brota de los avatares de la historia, del proceso de decantación de la moralidad humana en el que los principios se han venido estableciendo como tales y han obtenido su valor y distintividad, y en virtud de lo cual se legitima en la actualidad una adhesión irrestricta a su fuerza vinculante. Por la misma razón, en un modelo como este no resulta posible acometer la hermenéutica necesaria y suficiente que conduzca al significado profundo de esos principios, las razones que fundamentan efectivamente su jerarquía y el valor intransable o innegociable de algunos de ellos.

Teniendo en consideración la necesidad de sobrepasar estas limitaciones y fortalecer una orientación de mayor significación para la moralidad, la perspectiva de los derechos humanos es la construcción histórica capaz de resguardar de mejor manera ese conjunto intransable de valores y principios. Sólo desde la óptica de los derechos humanos se alcanza una orientación lúcida para conducir la interacción social en el sentido de lo que debemos y no debemos hacer a otros, de lo que tenemos que reconocer como límites de nuestra esfera de acción personal o social y de lo que debemos admitir como compromiso y deber para contribuir a que la vida pueda ser vivida por todos y cada uno de manera auténticamente humana.

No es otro el sentido presente en la Declaración sobre Bioética y Derechos Humanos(14), cuando ésta tiene en cuenta que los adelantos de la ciencia y la tecnología afectan tanto a nuestra comprensión de la vida como a la vida misma y, por ello, traen consigo una fuerte demanda de respuesta a los problemas éticos que suscitan; a la vez, reconoce que tales problemas deben examinarse teniendo en cuenta no sólo el respeto debido a la dignidad de la persona humana sino también el respeto 
universal y la observancia de los derechos humanos y las libertades fundamentales.

Se podría interpretar la decisión administrativa adoptada por la autoridad sanitaria de Chile -más que como una cuestión en la que se canalizan o defienden concepciones de vida buena, ideales de felicidad o sentidos de la virtud humana, es decir, nociones de máximos de moralidad-como la pretensión de establecer el resguardo de derechos fundamentales de las personas, al modo de un verdadero deber de Estado.

El sentido fundamental de la justicia, o el deber perfecto de la justicia -si quisiéramos indicarlo de ese modo- conmina a las autoridades a disponer medidas administrativas en las que se cautelen, entre otros, los derechos sexuales y reproductivos de las personas, en igualdad de oportunidades y acceso a los recursos que permiten ejercerlos más plenamente. La suscripción de los múltiples pactos internacionales obliga al Estado de Chile a establecer una atención preferencial por las cuestiones de justicia social, más todavía cuando ellas aluden a la salud pública y buscan conferir idénticas oportunidades a las personas, para corregir las enormes desigualdades en la distribución del riesgo reproductivo y los embarazos no deseados.

\section{Referencias}

1. Jiles J. De la miel a los implantes. Historia de las politicas de regulación de la fecundidad en Chile. Santiago de Chile: CORSAPS; 1992.

2. World Health Organization. Fact Sheet. March 2005 (versión original). Instituto Chileno de Medicina Reproductiva (ICMER). Levonorgestrel para anticoncepción de emergencia. Boletín informativo 2005 (versión: Unidad de Traducción ICMER, con permiso de OMS).

3. Casas L, Ortiz I. Anticoncepción de emergencia: Un marco jurídico. Santiago de Chile: SRE; 1997.

4. Convención sobre la eliminación de todas las formas de discriminación contra la mujer. Adoptada y abierta a la firma y ratificación, o adhesión, por la Asamblea General de ONU en su resolución 34/180, del 18 de diciembre de 1979. Entrada en vigor: 3 de septiembre de 1981.

5. Ministerio de Salud. Normas y Guía Clínica para la Atención en Servicios de Urgencia de Personas Victimas de Violencia Sexual. Santiago de Chile: MINSAL; 2004.

6. Ministerio de Salud. Instituto Chileno de Medicina Reproductiva (ICMER). Asociación Chilena de Protección de la Familia (APROFA). Normas técnicas y Guías clínicas sobre Regulación de la fertilidad. Santiago de Chile; 2006.

7. López Trujillo A, (Cardenal) Sgreccia E. (Monseñor) Sexualidad humana: Verdad y significado. Orientaciones educativas en familia. Ciudad del Vaticano: Pontificio Consejo para la Familia; 1995.

8. Ventura-Juncá P, Oyarzún E, Barros C. Evidencias cientificas disponibles sobre el efecto abortivo del Levonorgestrel (LNG) o píldora del día después. Santiago de Chile: Pontificia Universidad Católica de Chile; 2004.

9. Beauchamp TL, Childress JF. Principles of Biomedical Ethics. Oxford: Oxford University Press; 1994.

10. Gracia D. Ética y Vida. Estudios de Bioética. Santafé de Bogotá: Editorial El Búho; 1998.

11. Ross DW. The Right and the Good. Oxford: Oxford University Press; 2002.

12. Gracia D. Procedimientos de decisión en ética clinica. Madrid: EUDEMA; 1995.

13. Brussino S. Antecedentes y Fundamentos de la Ética de la Investigación (Material de trabajo del Curso de Introducción a la Ética de la Investigación en Seres humanos de la Red Bioética de UNESCO). 2006. (Sitio en Internet) Disponible en http://www.redbioetica-edu.com.ar

14. Declaración sobre Bioética y Derechos Humanos. Adoptada por aclamación el 19 de octubre de 2005 en la 33a sesión de la Conferencia General de UNESCO.

Recibido: 3 de julio de 2007.

Aceptado: 6 de agosto de 2007 . 